\title{
Mantle cell lymphoma and chronic lymphocytic leukemia: report of a rare disease association and review of the literature
}

\author{
Joana Perdigão $\cdot$ Helena Alaiz • Paulo Lúcio • \\ Paula Gameiro • Marta Sebastião • Lara Neto • \\ Maria Gomes da Silva $•$ José Cabeçadas
}

Received: 15 March 2010 / Accepted: 15 June 2010 /Published online: 7 July 2010

(C) Springer-Verlag 2010

\begin{abstract}
We report the case of an 86-year-old female who presented with lymphadenopathy, lymphocytosis, and thrombocytopenia. Peripheral blood immunophenotypic analysis identified two lymphoid B cell populations that differ in CD23, CD200, and CD43 expression and in the intensity of CD5, CD20, CD79b, and kappa light chain. By fluorescence in situ hybridization (FISH) analysis, approximately $30 \%$ of the mononuclear cells were positive for the $\mathrm{t}(11 ; 14)(\mathrm{q} 13 ; \mathrm{q} 32)$ and $40 \%$ for trisomy 12 ; analysis of the two populations separated by fluorescence activated cell sorting showed that $\mathrm{t}(11 ; 14)$ occurs exclusively in CD23-/CD200-/CD43- cells and trisomy 12 in the CD23+/CD200+/CD43+ population. Lymph node biopsy showed a typical small lymphocytic lymphoma morphology, with an effaced architecture and a predominantly diffuse proliferation of small to medium-sized lymphoid cells and pseudo-follicles with the characteristic features of proliferation centers. In addition, a different type of randomly dispersed nodules was present, composed of cells slightly larger than the monomorphic lymphocytic proliferation that occupied most of the lymph node. Staining
\end{abstract}

J. Perdigão $\cdot$ H. Alaiz $\cdot$ P. Lúcio $\cdot$ P. Gameiro $\cdot$ M. Sebastião $\cdot$

L. Neto $\cdot$ M. Gomes da Silva $\cdot$ J. Cabeçadas

Instituto Português de Oncologia de Lisboa Francisco Gentil,

Lisboa, Portugal

J. Perdigão $\cdot$ P. Lúcio · P. Gameiro • M. Gomes da Silva •

J. Cabeçadas

CEDOC, Faculdade de Ciências Médicas,

Universidade Nova de Lisboa,

Lisboa, Portugal

J. Perdigão $(\square)$

Laboratório de Hemato-Oncologia do Instituto Português de

Oncologia de Lisboa Francisco Gentil,

Rua Professor Lima Basto,

1099-023 Lisboa, Portugal

e-mail: jperdigao@ipolisboa.min-saude.pt for Cyclin D1 and FISH for CCND1 showed protein expression and gene breaks restricted to these areas. Conventional cytogenetic analysis revealed two separate clones, one with trisomy 12 and the other with $\mathrm{t}(11 ; 14)$ (q13;q32), both as unique clonal abnormalities. IGH analysis supports the presence of two populations with a different clonal origin. Taken together, these results provide strong evidence for the presence of a composite small lymphocytic lymphoma/chronic lymphocytic leukemia and mantle cell lymphoma. This association has been described in very rare cases that we briefly review.

Keywords Mantle cell lymphoma Small lymphocytic lymphoma/chronic lymphocytic leukemia . Composite lymphoma $\cdot$ Cytogenetic analysis

\section{Introduction}

Although small lymphocytic lymphoma/chronic lymphocytic leukemia (SLL/CLL) and mantle cell lymphoma (MCL) are well-defined clinical and biological entities easily distinguishable in most cases, some overlapping in the clinical, morphologic, and phenotypic features exist between these clonal CD5+ B cell lymphoproliferative disorders. In a proportion of cases, mainly when patients present with lymphocytosis without enlarged lymph nodes or when a lymph node biopsy cannot be obtained, this may result in a difficult differential diagnosis. The distinction is, however, clinically relevant since the expected course and treatment choices for these two neoplasms are essentially different.

Globally, CLL has an indolent course but the median survival can be highly variable, with some patients stable for several years and others progressing rapidly, with survival 
rates inferior to $1-2$ years. The disease is characterized by an absolute lymphocytosis in the peripheral blood and frequent bone marrow involvement by small, mature lymphocytes with nearly normal morphology. In most cases, CLL lymphocytes have easily identified immunophenotypic characteristics when studied by flow cytometry: they express CD5 and CD23 and are strongly positive for CD19 and negative for CD10; low levels of CD20 and CD22 are usually present. A weak expression of monoclonal surface immunoglobulins is present, while the cells are commonly negative or weakly positive for $\mathrm{CD} 79 \mathrm{~b}$ [1].

Lymph nodes are frequently involved and invaded by a monotonous population of lymphocytes with the typical CLL morphology. This massive infiltration effaces the normal architecture and has a pseudo-nodular pattern. Histological examination in low magnification shows "pseudo-follicles", corresponding to the proliferation centers common in this disease. Their lighter appearance is caused by the presence of a continuum of small, medium, and large cells, including lymphocytes, prolymphocytes, and paraimmunoblasts. Although circulating CLL cells are usually quiescent, cells present in these proliferation centers can also be found in the bone marrow and are believed to constitute the proliferative component of the disease [2].

CLL has no pathognomonic cytogenetic markers, but distinct cytogenetic abnormalities can be detected in about $80 \%$ of the affected patients by fluorescence in situ hybridization (FISH) analysis [3]. The more common alterations are trisomy 12, del(13q14), del(17p13) (P53), and del(11q22-23) (ATM). The finding of any of these abnormalities has a significant prognostic impact [3] and supports the diagnosis of CLL in the adequate clinical and morphological setting.

MCL is a relatively rare and aggressive B cell neoplasm with a median survival of 3-5 years [4]. Extranodal involvement is common, and a significant number of patients show extensive bone marrow and peripheral blood infiltration, with marked lymphocytosis. MCL lymphocytes are small to medium sized, with an irregular nuclear membrane, inconspicuous nucleoli, and variable amounts of cytoplasm, resembling centrocytes. Like CLL, MCL lymphocytes express CD5 and CD19. However, the strong expression of $\mathrm{CD} 79 \mathrm{~b}$ and $\mathrm{CD} 20$, and the moderate to intense surface Ig staining, as well as lack of $\mathrm{CD} 23$, are helpful for the differential diagnosis with CLL [5]. As recently described, CD200 seems to be a promising marker expressed by CLL, but not MCL cells that might be useful in the distinction of the two diseases [6].

The pattern of lymph node infiltration in MCL can be diffuse, nodular or limited to the follicular mantle zone. The nodular MCL pattern is very different from the one found in CLL: as opposed to CLL pseudo-follicles, MCL shows true follicles and is composed of a monomorphic popula- tion of small cells resembling centrocytes with scattered macrophages and a visible hyalinized small vascular proliferation in the more common cases.

MCL is genetically characterized by the presence of a $\mathrm{t}(11 ; 14)(\mathrm{q} 13 ; \mathrm{q} 32)$ translocation juxtaposing the genes $C C N D 1$ and $I G H$, which can be detected in approximately $97 \%$ of cases $[7,8]$. This gene rearrangement brings the $C C N D 1$ gene under the influence of the $I G H$ enhancer, causing the overexpression of cyclin D1 that can be detected in most cases by immunohistochemistry. Although rare cyclin D1-negative MCL cases have been described, this is still one of the most useful markers for the diagnosis of the disease.

The occurrence of two B cell lymphoproliferative disorders in the same patient has been reported rarely, either concomitantly or sequentially. Among these cases, composite lymphomas (with an incidence of 1-4\%) constitute a particular case of synchronous lymphomas, in which two non-Hodgkin lymphomas (NHL) or, more rarely, NHL and Hodgkin lymphoma are detected in the same anatomical compartment [9].

We present the case of a patient with a composite lymphoma, presenting with SLL/CLL and MCL at the time of diagnosis in peripheral blood and lymph nodes. Although the concomitant identification of each of these $B$ cell neoplasms and other lymphoid tumors has been described, the synchronous occurrence of both in the same patient has been reported in only a few cases. More importantly, since these entities share morphological and phenotypic characteristics, the simultaneous presence of both in the same anatomical compartments can represent a diagnostic challenge, that however has relevant clinical implication. This case also highlights the importance of a lymph node biopsy in cases where the diagnosis is not clear, as the tumors can be readily distinguished on morphological examination.

\section{Materials and methods}

\section{Peripheral blood smear}

Circulating lymphocyte morphology was assessed after staining of the peripheral blood smears with May-Grunwald-Giemsa, using a conventional optical microscope.

Histology and immunohistochemistry

After fixation in neutral buffered formalin, the lymph node was embedded in paraffin and sectioned following routine methods. Sections were stained with H\&E. Immunohistochemical staining with anti-CD20, PAX5, CD10, CD5 CD23, BCL-2, CD3, CD21, and cyclin D1 antibodies was performed according to a standardized automated protocol. 
Flow cytometry

Multiparameter flow cytometry was performed on an EDTA-anticoagulated peripheral blood (PB) sample. Erythrocyte lysis, stain, and washing were done according to well-established procedures as described [10]. Seven-color monoclonal antibody surface staining was performed. CD103-FITC, CD22-PE, CD11c-PE, CD5-PERCP-CY5.5, CD19- PERCP-CY5.5, CD3-PERCP-CY5.5, CD10-APC, CD23-APC, CD25-APC, and CD43-APC H7 were obtained from Becton Dickinson Biosciences (San Jose, CA, USA); LAMBDA-FITC, CD79b-FITC, and KAPPAPE from DakoCytomation (Glostrup, Denmark); CD19-PECy7 and CD5-PE-CY7 from Immunotech (Marseille, France); CD38-AlexaFluor700 and CD45-AlexaFluor700 from EXBIO Praha (Vestec u Prahy, Czech Republic); CD20 PACIFIC BLUE and CD200-APC from eBiosciences (San Diego, CA, USA). Data acquisition and analysis were performed in a FACSCanto II flow cytometer using the Infinicyt software (Cytognos, Salamanca, Spain). 100,000 events were acquired for each combination.

Fluorescence activated cell sorting

The B cell populations were separated by fluorescence activated cell sorting (FACS) sorting using a FACS ARIA cell sorter, BD Biosciences. Separation was based on the distinct CD200 and CD23 expression. The purity of the sorted populations was $99.8 \%$ and $96.5 \%$ for the CLL and MCL populations, respectively (Fig. 1). Sorted cells were used for FISH analysis as described below.

Conventional cytogenetics and fluorescence in situ hybridization studies

Conventional cytogenetic studies were performed in Gbanded chromosome preparations by standard methods. Metaphases were obtained after 72-h stimulated cultures of a lymph node cell suspension.

FISH was performed in peripheral blood mononuclear cells and in sorted B cell populations using the following commercially available probes, according to the manufacturer's instructions: t $(11 ; 14)$ dual-fusion (LSI IGH SpectrumGreen / LSI CCND1 SpectrumOrange), LSI p53, LSI ATM, LSI D13S319, LSI 13q34, and CEP12 (LSI p53/LSI ATM and LSI D13S319/LSI 13q34/CEP12 Multi-color Probe Sets). All probes were obtained from Abbott Molecular Inc., Des Plaines, IL. After hybridization and washes, preparations were counterstained with DAPI (4,6-diamidino-2-phenylindole) and slides were examined using a fluorescence microscope equipped with appropriate filter sets. Two hundred interphase nuclei were scored for each probe. For hybridization in metaphases, slides were first G-banded in order to identify and localize metaphase spreads with the $\mathrm{t}(11 ; 14)$. These slides were then de-stained with Carnoy solution and used for FISH using standard methods. Cut-off values for the used probes are: $\mathrm{t}(11 ; 14)$ dual-fusion, $0 \%$; LSI p53, 5.8\%; LSI ATM, 4.4\%; LSI D13S319, 4.4\%; LSI 13q34, 4.0\%; and CEP12, 1.6\%.

Sections of the lymph node were hybridized with the CCND1 FISH DNA probe, Split Signal, using the Histology FISH Accessory Kit, both from Dako A/S, Denmark, as prescribed in the manufacturer's instructions.

IGH and IGK rearrangement analysis

Clonality analysis was done in $\mathrm{PB}$ and lymph node snap-frozen biopsy samples obtained at time of diagnosis. DNA was extracted using the Gentra Puregene Cell Kit (QIAGEN, Maryland, USA). DNA isolated from both samples showed amplification up to 600 base pairs (bp) of the BIOMED-2 control gene polymerase chain reaction $(\mathrm{PCR})$ [11]. Immunoglobulin gene (Ig) rearrangements were studied using the BIOMED-2 multiplex PCR primers and protocols [11]: $I G H\left(\mathrm{~V}_{\mathrm{H}^{-}} \mathrm{J}_{\mathrm{H}}\right.$ and $\left.\mathrm{D}_{\mathrm{H}^{-}} \mathrm{J}_{\mathrm{H}}\right)$ and $I G K\left(\mathrm{~V}_{\mathrm{k}}-\mathrm{J}_{\mathrm{k}}\right.$ and $\mathrm{Kde}$ rearrangements) analysis were performed. Each PCR reaction was done in duplicate, with $100 \mathrm{ng}$ of DNA. PCR products were evaluated by GeneScan fragment analysis (ABI PRISM 310 and 3130 Genetic Analyzers, Applied Biosystems, CA, USA). Sequence of $\mathrm{V}_{\mathrm{H}^{-}}-\mathrm{D}_{\mathrm{H}^{-}} \mathrm{J}_{\mathrm{H}}$ rearrangements was done using ABI BigDye Terminator v1.1 Cycle Sequencing Kit (Applied Biosystems, CA, USA). Mutational status analysis was based in IMGT database (http://imgt.cines. fr) and followed ERIC recommendations [12].

\section{Results}

Case report

An 86-year-old caucasian woman was referred to the Hematology clinic in January 2009 for evaluation of lymphocytosis and enlarged cervical lymph nodes that had been present for a few months. She complained of fatigue and her medical history revealed the presence of Alzheimer's disease, elevated blood pressure, and arthritis.

The clinical exam confirmed the presence of multiple, small (less than $2.5 \mathrm{~cm}$ maximum diameter) bilateral cervical and axillary lymph nodes. No other adenopathies or enlargement of the spleen or liver was palpable. A whole body CT scan showed the presence of mediastinal and retroperitoneal non-bulky adenopathies (max diameter $4 \mathrm{~cm}$ ) and a slightly enlarged but homogeneous liver.

Peripheral blood counts showed a hemoglobin of $11.7 \mathrm{~g} / \mathrm{dl}$, hematocrit of $34 \%$, a white blood cell (WBC) 
A
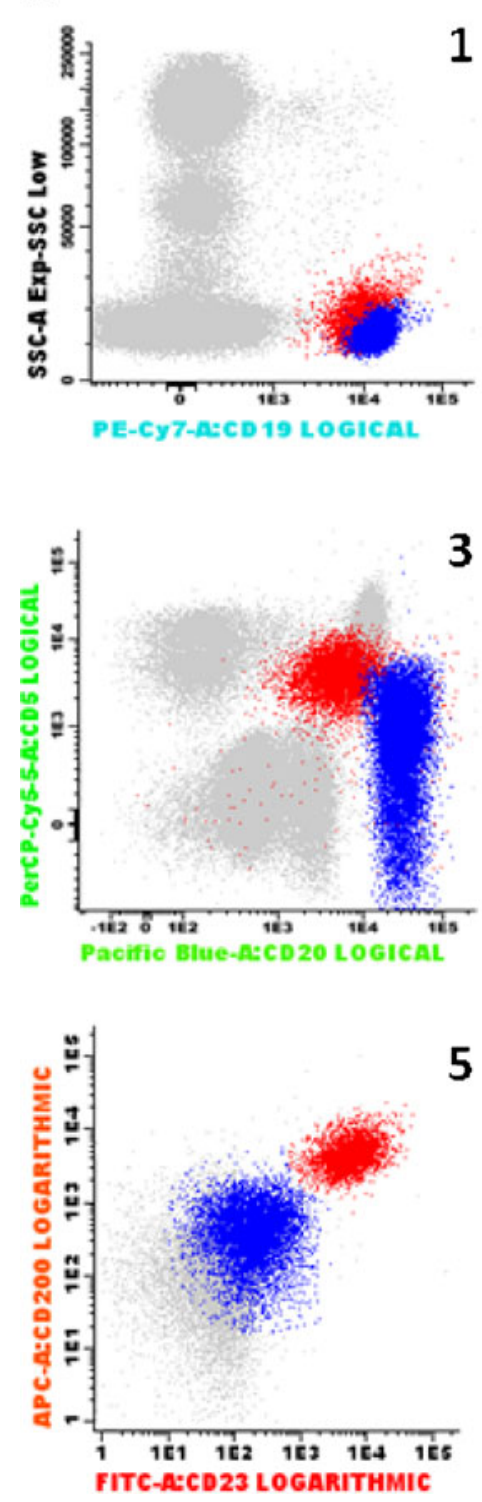

Fig. 1 Immunophenotype of peripheral blood B cells and sorted populations. a Peripheral blood sample showing two clonal B cell populations, identified as CD19+/kappa+ cells (dot-plots 1 and 2). Population depicted in red has a typical CLL phenotype, expressing CD5 and dim kappa light chains, CD20 and CD79b. These cells also expressed strong CD200, CD23, and CD43. The

count of $42,150 / \mu 1$ (with $91 \%$ lymphocytes), and a platelet count of $125,000 / \mu 1$. Lymphocyte morphology was typical of CLL except for the presence of $4 \%$ of large cells with nucleolated, immature chromatin and moderately basophilic cytoplasm.

Phenotypic characterization of peripheral blood lymphocytes by flow cytometry revealed the presence of two distinct CD5+ B cell populations. FISH analysis of PB mononuclear cells showed the presence of $t(11 ; 14)$ and trisomy 12 in different proportions of cells, also suggesting the occurrence of two neoplastic cell populations (see below).
B
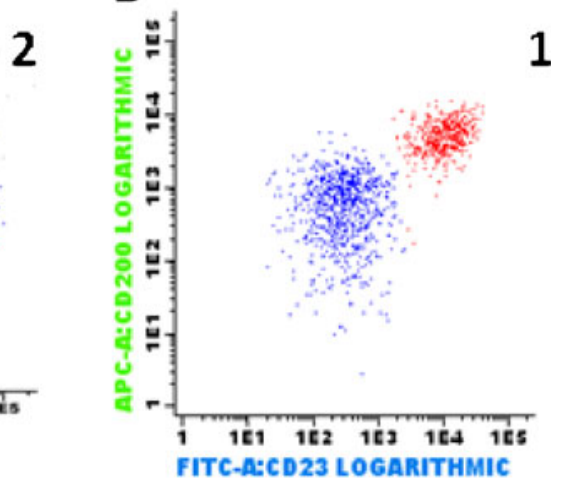

4
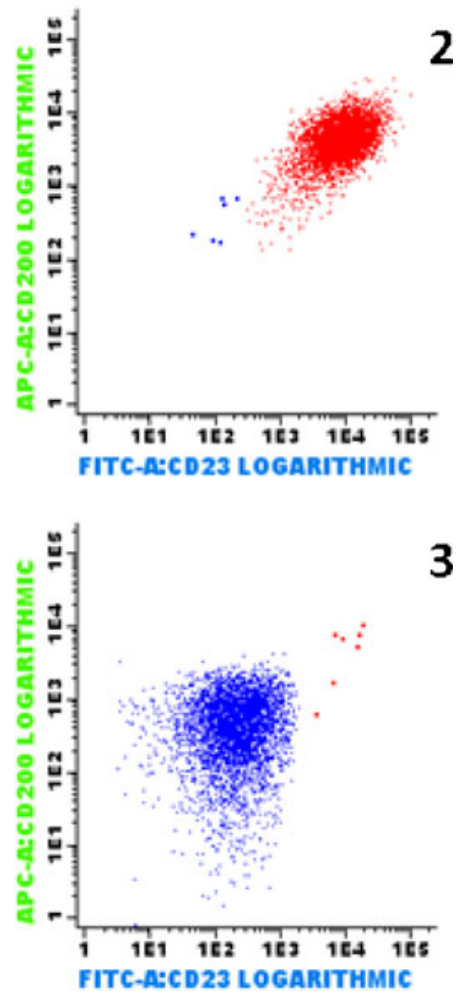

neoplastic B cells represented in blue can be distinguished by the stronger light chain, CD20 and CD79b expression and a dimmer or negative expressions of CD5, CD200, CD23, and CD43. b The two B cell populations were separated by FACS with very high efficiency. Dot-plot 1 shows the pre-sorting analysis; 2 and 3 show analysis of the sorted populations (same colors as in A)

A lymph node biopsy was performed, and a diagnosis of composite SLL/CLL and stage IV-A mantle cell lymphoma was made.

Due to a rapid WBC count increase and worsening of anemia, palliative oral chemotherapy with monthly cycles of Chlorambucil (1mg/kg/cycle) and Prednisone $(1 \mathrm{mg} / \mathrm{kg} /$ day $\times 5$ days) was started. The choice of this regimen, more appropriate for CLL than MCL, was dictated by the advanced patient age and the non-curative perspective of the treatment. After four cycles, fatigue persisted but WBC and lymphocyte counts were significantly reduced (WBC 
$7,580 / \mu 1$ with $65 \%$ lymphocytes), and cervical lymph nodes are no longer detectable. Treatment was stopped after 12 cycles and at 16 months follow-up the patient is asymptomatic, with normal lymphocyte counts and small, stable lymph nodes. The two circulating clonal B lymphocyte populations are still detectable by flow cytometry and FISH.

\section{Immunophenotyping}

Flow cytometric analysis was performed on $\mathrm{PB}$, with gating on CD45-bright lymphocytes. Seventy-seven percent of monoclonal B lymphocytes (CD19+, CD22+, CD10- and restricted expression of kappa light chains) were detected. Within these, two cell populations were identified that could be distinguished by the positivity for CD23, CD200, and CD43 and the levels of expression of CD20, CD5, and $\mathrm{k}$ light chains. The CD23/CD200/CD43-positive population (57.6\% of B lymphocytes) showed brighter CD5 staining and weaker CD20, kappa and CD79b, in contrast to the CD23/CD200/CD43-negative population (42.4\%) (Fig. 1).

In fact, the $\mathrm{CD} 23+/ \mathrm{CD} 200+/ \mathrm{CD} 43+$ population had a typical CLL immunophenotype, as defined by the IWCLLWG [1]: co-expression of CD5, CD19, CD23, low CD20, low sIg, and low CD79b. On the other hand, the phenotype of the CD23-/CD200-/CD43- population suggested the possible diagnosis of MCL.

\section{Histopathologic analysis}

The lymph node architecture was effaced by a population of small to medium-sized lymphocytes organized in a predom- inantly diffuse pattern with some nodular structures. The predominant population in the diffuse areas consisted of small, mature lymphocytes with scarce cytoplasm and round nuclei with condensed chromatin. These cells stained positively for CD20, CD5, and CD23. Two types of nodular structures were present: the paler nodules were composed of medium-sized cells with abundant prolymphocytes and paraimmunoblasts, recognizable as proliferation centers. The darker nodules were composed of small to medium-sized cells, with slightly irregular nuclear membrane and morphology similar to centrocytes. Immunohistochemistry studies showed that these areas corresponded to a different cellular population that stained with $\mathrm{CD} 20, \mathrm{CD} 5$, and Cyclin D1, but not CD23 (Figs. 2, 3). Also, absence of CD21+ cells excluded the possibility of these being remnants of germinal centers. FISH analysis in lymph node sections using a CCND1 probe showed the presence of gene breaks exclusively in cells present in these nodules.

Some cells interspersed in the CLL-looking areas also showed positivity for Cyclin D1 (Fig. 3).

\section{Conventional and molecular cytogenetics analysis}

FISH analysis was performed in PB mononuclear cells using a dual-fusion/dual-color $\mathrm{t}(11 ; 14)(\mathrm{q} 13 ; \mathrm{q} 32)$ probe and the panel of probes that is routinely employed in a CLL diagnostic work-up: p53 (17p13.1); ATM (11q22.3); D13S319 (13q14.3); 13q34 and CEP12 (12p11.1-q11). No deletions of the p53,13q14, 13q34, and ATM region were detected. Forty-one percent of the nuclei showed three signals for CEP 12 and $33 \%$ were scored as positive for $\mathrm{t}(11 ; 14)$ although with an atypical pattern: one fusion signal
Fig. 2 In hematoxylin-eosin staining of lymph node section, the MCL compartment is discernible as a nodular aggregate of smaller, darker cells in the center of the left panel. In the right panel, the border between the two components is visible at higher magnification
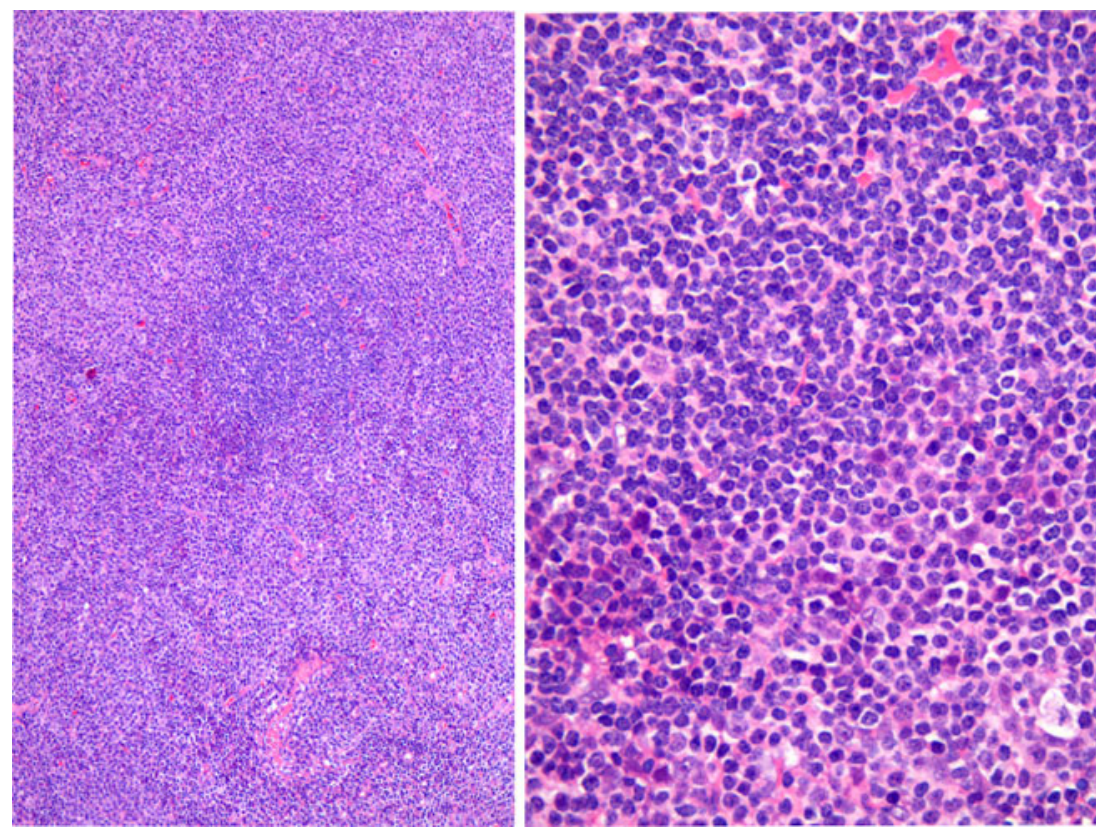
Fig. 3 Immunohistochemical staining for cyclin D1 highlights the MCL component in the lymph node $(\mathbf{a}, \mathbf{b})$ and CD23 staining shows the CLL/SLL cells in a reverse pattern $(\mathbf{c}, \mathbf{d})$. $\mathrm{A}$ and $\mathrm{C}$ at low magnification; $\mathrm{B}$ and $\mathrm{D}$ at medium power
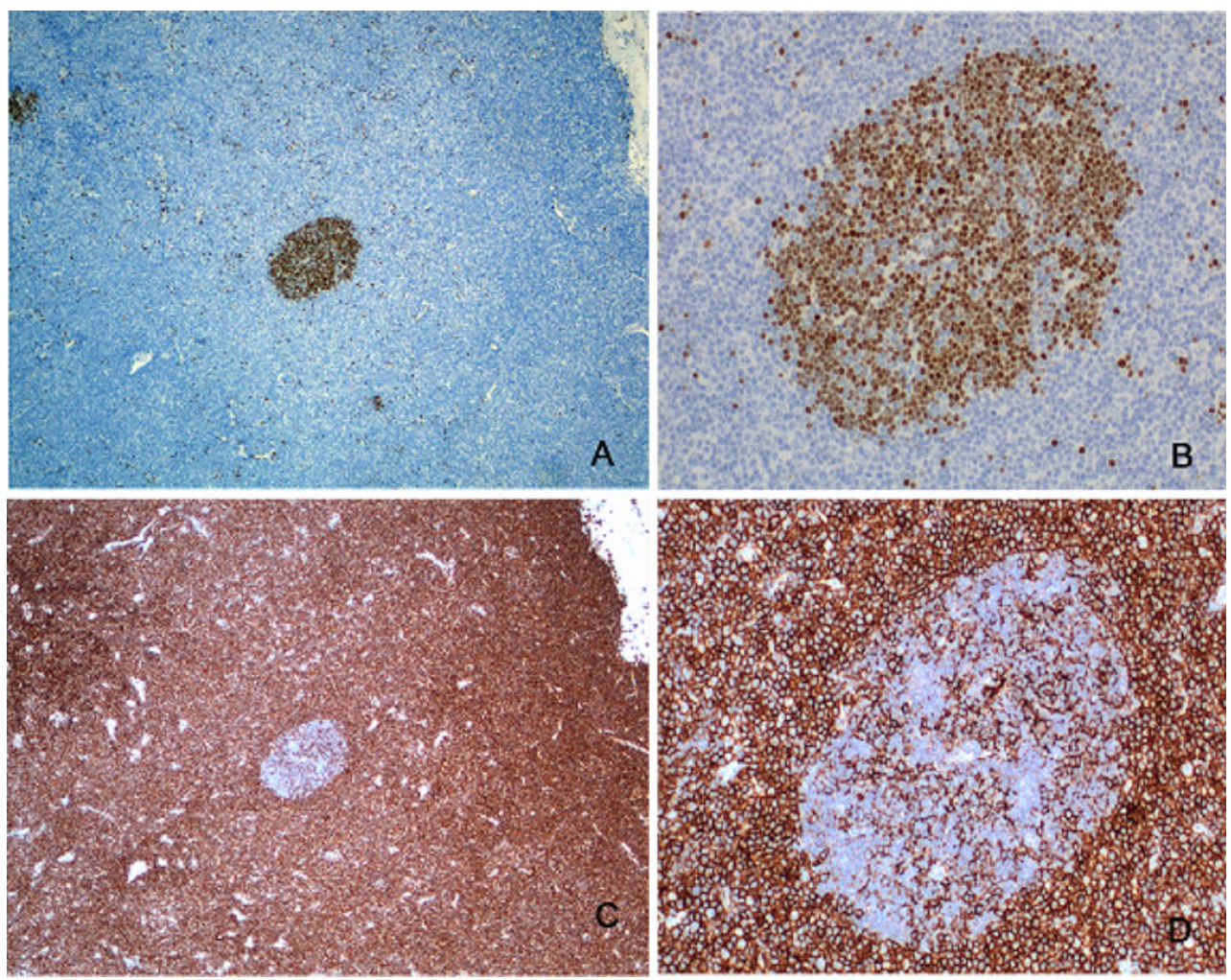

plus one green (LSI IGH) and two red (LSI CCND1) signals. This result indicates a loss of genetic material corresponding to the IGH region in one of the derivative chromosomes. The observed difference in the percentage of genetically rearranged cells with the two probes suggested the presence of two different clones. This pattern could be interpreted as indicating the presence of two independent cell clones, each containing a unique genetic abnormality. In contrast, a subclone having acquired an extra chromosome 12 could have evolved from the original $t(11 ; 14)$ positive clone.

The presence of two independent clones, one with trisomy 12 and the other with $\mathrm{t}(11 ; 14)$ (q13;q32), was confirmed by conventional cytogenetic analysis of the lymph node biopsy (Fig. 4). Furthermore, FISH analysis in the two immunophenotypically distinct populations separated by sorting showed trisomy 12 to be restricted to the $\mathrm{CD} 23+/ \mathrm{CD} 200+$ population (CLL cells positive for trisomy $12(95.2 \%)$ and negative for $\mathrm{t}(11 ; 14))$ and $\mathrm{t}(11 ; 14)$ restricted to the $\mathrm{CD} 23-/ \mathrm{CD} 200-$ cells (MCL population positive for $\mathrm{t}(11 ; 14)(100 \%)$ and negative for Trisomy 12). These results confirm the presence of two diseases.

In order to clarify the atypical pattern previously observed in interphase FISH with the $\mathrm{t}(11 ; 14)$ probe, FISH was repeated with the same probe in selected metaphases where the $t(11 ; 14)$ translocation was cytogenetically identified. In these, it could be observed that the only fusion signal detected corresponded to the der(14) chromosome, with the der(11) chromosome missing the IGH fragment. This result is consistent with the overexpression of Cyclin D1 due to activation of the CCND1 caused by its relocation to the der(14) chromosome [13]

\section{IGH and IGK rearrangement analysis}

$I G H$ analysis detected three $I g$ gene rearrangements in the PB DNA sample: two complete $\left(\mathrm{V}_{\mathrm{H}}-\mathrm{D}_{\mathrm{H}}-\mathrm{J}_{\mathrm{H}}\right)$ and one incomplete $\left(\mathrm{D}_{\mathrm{H}^{-}} \mathrm{J}_{\mathrm{H}}\right)$ (Fig. 5). The presence of three $I G H$ rearrangements strongly suggests the existence of two clones since in each allele, only one $I G H$ rearrangement can be detected using this PCR strategy [11]. This is further supported by the fact that none of the two populations have an extra $I G H$ gene copy (on the contrary, the MCL cells have just one, as shown by FISH). In the lymph node DNA, the incomplete $\mathrm{D}_{\mathrm{H}^{-}} \mathrm{J}_{\mathrm{H}}$ rearrangement was not detected (Fig. 5).

$I G K$ analysis revealed the presence of one (and the same) $\mathrm{V}_{\mathrm{k}}-\mathrm{J}_{\mathrm{k}}$ rearrangement in both the lymph node and peripheral blood DNA samples. Regarding Kde, two rearrangements were detected in the PB DNA, but only one in the lymph node.

Non-detection of one $I G H$ and one Kde rearrangement in the lymph node sample can be due to the presence of a smaller amount of DNA belonging to one of the two B cell clones, most probably the MCL one that has a minor representation in this tissue (Figs. 2, 3). 


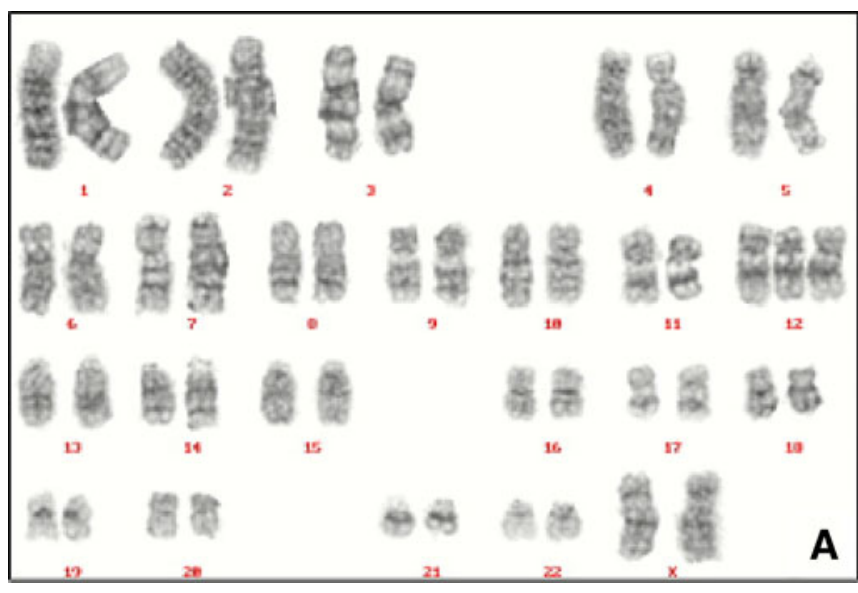

Fig. 4 Conventional cytogenetic analysis of the lymph node biopsy revealed the presence of two independent clones. Description of the karyotype (ISCN 2009): 46,XX,t(11;14)(q13;q32)[9]/47,XX,+12[3]/

Regarding $\mathrm{V}_{\mathrm{k}}-\mathrm{J}_{\mathrm{k}}$, the fact that only one rearrangement was detected in both DNA samples although both clones were shown to express a Kappa chain can be due to the under-representation of the MCL clone DNA on both samples together with the preferential amplification of one of the $\mathrm{V}_{\mathrm{k}}-\mathrm{J}_{\mathrm{k}}$ rearrangements. Alternatively, the detection of a unique $\mathrm{V}_{\mathrm{k}}-\mathrm{J}_{\mathrm{k}}$ rearrangement due to impairing of primer annealing caused by somatic hypermutation cannot be excluded.

Sequencing of the two complete $I G H$ gene rearrangements showed that both were in-frame, one mutated $(94.2 \%$ homology) and the other non-mutated. Different segment usage was observed in both rearrangements: IGHV3-74/ IGHD2-15/IGHJ4 in the mutated allele and IGHVI-18/ IGHD6-19/IGHJ4 in the non-mutated one. The particular segments used in the two rearrangements and the different

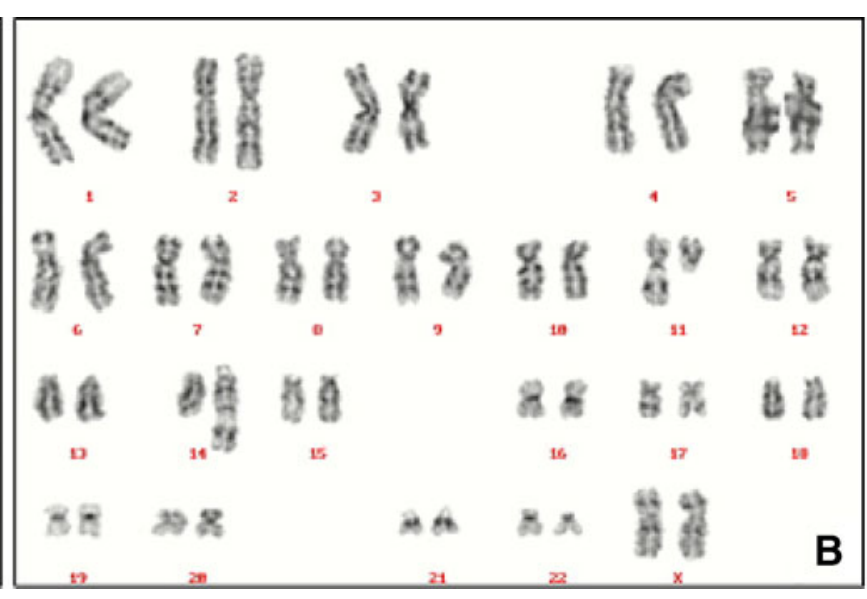

46,XX[2]. a Karyogram of the Trisomy 12 clone. b Karyogram of the $\mathrm{t}(11 ; 14)(\mathrm{q} 13 ; \mathrm{q} 32)$ clone

CDR3 sequences obtained (data not shown) support the hypothesis of a different clonal origin.

\section{Discussion}

The differential diagnosis between MCL and SLL/CLL, both with frequent leukemic presentations, usually relies in the phenotypic characteristics of the small, B CD5+ circulating lymphocytes. There are, however, difficult borderline cases in which a detailed histopathologic analysis is essential for a correct diagnosis and the presence of distinct cytogenetic alterations can provide important information [14].

Furthermore, it should be kept in mind that, although rare, the occurrence of two different lymphomas in the

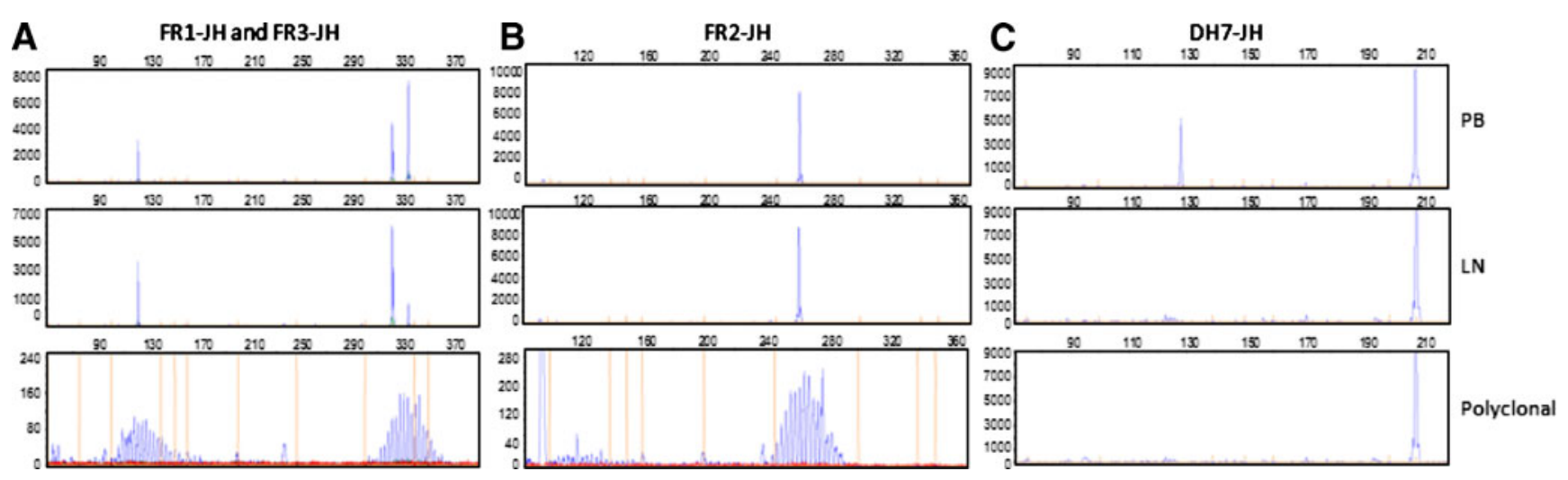

Fig. $5 I G H$ clonality profiles from the peripheral blood and lymph node samples obtained at diagnosis. Polyclonal controls were obtained from tonsil DNA. Genescan results using the BIOMED2 protocols: $I G H$ tube A-FR1 and tube C-FR3 (A), tube B-FR2 (B) and tube E-DH7. a Two clonal peaks are detected with FR1 primers (334 bp and $322 \mathrm{bp}$ ) in both the peripheral blood $(P B)$ and lymph node $(L N)$ DNA samples. FR3 primers only detected one rearrange- ment $(121 \mathrm{bp})$ in both samples. This can be due to the presence of somatic hypermutation in one of the alleles (see text). b Only one gene rearrangement $(260 \mathrm{bp})$ is detected in both the $P B$ and $L N$ samples. Again, most probably the presence of somatic hypermutation has hampered PCR amplification in one of the alleles. c One incomplete gene rearrangement (DH7-JH, $128 \mathrm{bp}$ ) was detected only in the $P B$ 
same patient has been widely recognized. In these cases, the investigation of the clonal relatedness of the two populations is important for the characterization of the involved diseases: while the demonstration of a clonal relationship implies that one of the diseases evolved from the other, the contrary implies a distinct origin of the two clones. In addition to the contribution to understanding the biology of the diseases, this distinction is important since the "transformation" to a more aggressive type of disease, as opposed to the independent development of both, is usually associated with a poorer prognosis.

To the best of our knowledge, only seven cases of the simultaneous occurrence of MCL and SLL/CLL have been previously described.

The case reported by Medlicott et al. describes a patient with MCL identified in the ileum and appendix and a CLL in the bone marrow [15]. Although both diseases occurred synchronously, this was not a composite lymphoma.

Sanchez et al. described four cases of synchronous BCLL and MCL, as part of a group of 53 patients with leukemic B cell chronic lymphoproliferative disorders in which two or more B cell clones could be identified based on morphological and phenotypic characteristics [16]. In three of them, the presence of two clones was demonstrated by $I G H$ rearrangement molecular studies. The remaining two cases $[17,18]$ are SLL/CLL-MCL composite lymphomas: in both, the two diseases could be identified concomitantly in $\mathrm{PB} /$ bone marrow and lymph nodes, and convincing proof of the presence of two clones is presented.

The case we present here is thus the third case of a biclonal composite SLL/CLL-MCL with lymph node and PB involvement by both diseases. Its description emphasizes the importance of a complete diagnostic work-up of suspected low-grade lymphomas, in particular involving the diagnosis of difficult CLL and/or MCL cases. In fact, atypical CLL and MCL cases have been described regarding morphology, surface marker expression, and genetic abnormalities [14]. For this reason, in some situations, not only CLL and MCL can be difficult to distinguish from each other, but also the presence of two diseases can be missed and data interpreted as the presence of a single, atypical disease.

In our case, the immunophenotypic differences between the two populations identified in PB are subtle, namely because the light chain expressed is the same. In the absence of a careful analysis of the expression levels of the several markers, it could have been interpreted as an atypical CLL with strong CD20 and Kappa expression. On the other hand, the finding of $\mathrm{t}(11 ; 14)$ could have led to the diagnosis of MCL.

The demonstration of the presence of both neoplasms in the lymph node was only possible by a detailed histopathologic analysis that identified two populations with the typical MCL and CLL phenotype in separate areas. Cyclin D1 staining was critical in allowing a clear identification of the lymph node nodules as corresponding to MCL and distinguishing them from the expected CLL proliferation centers; this observation was complemented by the demonstration of CCND1 gene breaks in the "MCL areas".

Finally, definite proof of biclonality was obtained by conventional cytogenetics in the lymph node with $t(11 ; 14)$ and trisomy 12 as unique abnormalities and the demonstration of the mutually exclusive presence of the two cytogenetic abnormalities in sorted populations. This was further supported by the presence of three $I G H$ gene rearrangements that is in agreement with a different clonal origin for both diseases.

It is worth noting that, in MCL, the detection of $\mathrm{t}(11 ; 14)$ as an isolated abnormality by conventional cytogenetic analysis is a rare situation (previously described in about $10 \%$ of the MCL cases in which an abnormal karyotype is detected [19]) that adds to the uniqueness of this case. This finding may be related to the somewhat indolent behavior observed; in fact, although treated with a non-aggressive regimen and showing persistence of both circulating disease clones, the patient is clinically stable 16 months after diagnosis.

In conclusion, it should be kept in mind that although in the vast majority of cases, the routine diagnosis of CLL can rely solely on immunophenotyping and FISH analysis of $\mathrm{PB}$ as recommended by the current guidelines of the International Workshop on CLL [1], in some situations a more refined diagnosis work-up may be necessary. The rare possibility of the simultaneous occurrence of SLL/CLL and MCL should be suspected in the presence of a cytological low-grade B cell malignancy with a leukemic presentation and a phenotype suggesting the presence of two clones. Furthermore, since the presence of $t(11 ; 14)$ is not compatible with the diagnosis of CLL according to the WHO 2008 classification, its detection by FISH in PB in the context of a suspected CLL should call attention to the need for a more detailed characterization, possibly involving histological analysis. The characterization of these difficult cases should always be done in specialized centers with expertise in the various approaches required for an accurate diagnosis, including morphological and histological analysis performed by an expert hematopathologist, imunophenotypic and genetic studies.

Acknowledgments We thank Ana Luísa Caetano for the separation of the two B cell populations by FACS sorting.

Conflict of interest The authors declare that they have no conflict of interest. 


\section{References}

1. Hallek M, Cheson BD, Catovsky D et al (2008) Guidelines for the diagnosis and treatment of chronic lymphocytic leukemia: a report from the International Workshop on Chronic Lymphocytic Leukemia updating the National Cancer Institute-Working Group 1996 guidelines. Blood 111:54465456

2. Caligaris-Cappio F, Ghia P (2008) Novel insights in chronic lymphocytic leukemia: are we getting closer to understanding the pathogenesis of the disease? J Clin Oncol 26:4497-4503

3. Dohner H, Stilgenbauer S, Benner A et al (2000) Genomic aberrations and survival in chronic lymphocytic leukemia. N Engl J Med 343:1910-1916

4. O'Connor OA (2007) Mantle cell lymphoma: identifying novel molecular targets in growth and survival pathways. Hematology $1: 270-276$

5. Craig FE, Foon KA (2008) Flow cytometric immunophenotyping for hematologic neoplasms. Blood 111:3941-3967

6. Palumbo GA, Parrinello N, Fargione G et al (2009) CD200 expression may help in differential diagnosis between mantle cell lymphoma and B-cell chronic lymphocytic leukemia. Leuk Res 33:1212

7. Belaud-Rotureau M-A, Parrens M, Dubus P et al (2002) A comparative analysis of FISH, RT-PCR, PCR, and immunohistochemistry for the diagnosis of mantle cell lymphomas. Mod Pathol 15:517-525

8. Remstein ED, Kurtin PJ, Buño I et al (2000) Diagnostic utility of fluorescence in situ hybridization in mantle-cell lymphoma. $\mathrm{Br} \mathrm{J}$ Haematol 110:856-862

9. Jaffe ES, Wilson WH (2004) Gray zone, synchronous, and metachronous lymphomas: diseases at the interface of Hodgkin's and non-Hodgkin's lymphomas. In: Mauch PM, Armitage JO, Coiffier B et al (eds) Non-Hodgkin's Lymphomas. Lippincott Williams \& Wilkins, Philadelphia, pp 69-80
10. Bueno C, Almeida J, Lucio P et al (2004) Incidence and characteristics of $\mathrm{CD}^{+} / \mathrm{HLA} \mathrm{DR}^{\text {hi }}$ dendritic cell malignancies. Haematologica 89:58-69

11. van Dongen JJM, Langerak AW, Brüggemann M et al (2003) Design and standardization of PCR primers and protocols for detection of clonal immunoglobulin and T-cell receptor gene recombinations in suspect lymphoproliferations: Report of the BIOMED-2 Concerted Action BMH4-CT98. Leukemia 17:2257-2317

12. Ghia P, Stamatopoulos K, Belessi C et al (2007) ERIC recommendations on IGHV gene mutational status analysis in chronic lymphocytic leukemia. Leukemia 21:1-3

13. Huret JL (1998) t(11;14)(q13;q32). In: Atlas Genet Cytogenet Oncol Haematol. Available at: http://AtlasGeneticsOncology.org/ Anomalies/t1114ID2021.html. Accessed March 8, 2010

14. Zhao XF (2009) Pitfalls in diagnostic hematopathology: part I. Int J Clin Exp Pathol 2:11-20

15. Medlicott SAC, Brown AH, Roland B et al (2007) Multiple lymphomatous diverticulosis and comorbid chronic lymphocytic leukemia: novel manifestations of ileocolic mantle cell lymphoma. Int J Surg Pathol 15:408-413

16. Sanchez M-L, Almeida J, Gonzalez D et al (2003) Incidence and clinicobiologic characteristics of leukemic B-cell chronic lymphoproliferative disorders with more than one B-cell clone. Blood 102:2994-3002

17. Fend F, Quintanilla-Martinez L, Kumar S et al (1999) Composite low grade B-cell lymphomas with two immunophenotypically distinct cell populations are true biclonal lymphomas. Am J Pathol 154:1857-1866

18. Lima M, Pinto L, Teixeira MDA et al (2003) Guess what: chronic $13 \mathrm{q} 14.3^{+} / \mathrm{CD} 5-/ \mathrm{CD} 23+$ lymphocytic leukemia in blood and $\mathrm{t}$ $(11 ; 14)(\mathrm{q} 13 ; \mathrm{q} 32)+/ \mathrm{CD} 5+/ \mathrm{CD} 23-$ mantle cell lymphoma in lymph nodes! Cytom B Clin Cytom 51B:41-44

19. Salaverria I, Espinet B, Carrió A et al (2008) Multiple recurrent chromosomal breakpoints in mantle cell lymphoma revealed by a combination of molecular cytogenetic techniques. Genes Chromosomes Cancer 47:1086 